\title{
What is the Value of the 60 Minute Cortisol Measurement in the Short Synacthen Test (SST)? Evidence for the Defence
}

\author{
Maria Michaelidou ${ }^{1}$, Ghasem Yadegarfar ${ }^{2}$, Lauren Morris ${ }^{1}$, Samantha Dolan ${ }^{1}$, Adam \\ Robinson $^{3}$, Asma Naseem ${ }^{1}$, Mark Livingston ${ }^{4}$, Christopher Duff ${ }^{5}$, Anthony Fryer ${ }^{6}$, Peter \\ Trainer $^{7}$, and Adrian Heald ${ }^{3}$ \\ ${ }^{1}$ Salford Royal NHS Foundation Trust \\ ${ }^{2}$ Isfahan University of Medical Sciences \\ ${ }^{3}$ Salford Royal Hospitals NHS Trust \\ ${ }^{4}$ Walsall Healthcare NHS Trust \\ ${ }^{5}$ University Hospitals of North Midlands \\ ${ }^{6}$ Keele University \\ ${ }^{7}$ Christie Hospital
}

March 23, 2021

\begin{abstract}
The Short Synacthen Test is the way that we most often determine whether people's adrenal glands are working. We here have shown that an extra blood sample taken at 60 minutes post Synacthen vs a 30 minute sample alone, may make the test more effective at excluding those people who do not need to go on hydrocortisone supplementation or need further evaluation.

What is the Value of the 60 Minute Cortisol Measurement in the Short Synacthen Test (SST)? Evidence for the Defence

Michaelidou $\mathrm{M}^{1 \S}$, Yadegarfar $\mathrm{G}^{2,3} \S$, Morris $\mathrm{L}^{1}$, Dolan $\mathrm{S}^{1}$, Robinson $\mathrm{A}^{1}$; Naseem $\mathrm{A}^{1}$; Livingston $\mathrm{M}^{4}$, Duff $\mathrm{CJ}^{5,6}$,

Fryer $\mathrm{AA}^{5,6}$, Trainer $\mathrm{P}^{7}, \mathrm{AH}$ Heald ${ }^{* 1,2}$

${ }^{1}$ Department of Endocrinology and Diabetes, Salford Royal Hospital, Salford, ${ }^{2}$ The School of Medicine and Manchester Academic Health Sciences Centre, University of Manchester; ${ }^{3}$ Isfahan University of Medical Sciences, Isfahan, Islamic Republic of Iran; United Kingdom ${ }^{4}$; Department of Clinical Biochemistry, Black Country Pathology Services, Walsall Manor Hospital, Walsall; ${ }^{5}$ Department of Clinical Biochemistry, University Hospitals of North Midlands NHS Trust, Stoke on Trent; ${ }^{6}$ School of Primary, Community and Social Care, Keele University; Department of Endocrinology; Christie Hospital, Manchester ${ }^{7}$

* Corresponding Author

$\S$ Joint first author
\end{abstract}

Key words: adrenal insufficiency, cortisol, short synacthen test

Corresponding author:

Dr Adrian H Heald 
Department of Diabetes and Endocrinology

Salford Royal Hospital

Salford

M6 8HD

Telephone +441612065157

Email: adrian.heald@manchester.ac.uk

Word Count (main text only): 596

\begin{abstract}
The Short Synacthen Test is the way that we most often determine whether people's adrenal glands are working.

We here have shown that an extra blood sample taken at 60 minutes post Synacthen vs a 30 minute sample alone, may make the test more effective at excluding those people who do not need to go on hydrocortisone supplementation or need further evaluation.
\end{abstract}

\title{
Letter to the editor
}

Adrenal insufficiency is caused by failure of the adrenal glands to produce physiological amounts of cortisol and sometimes aldosterone (in the case of Addison's Disease). Adrenal insufficiency can be a consequence of pituitary / adrenal insufficiency or suppression of endogenous cortisol production as a result of taking exogenous steroids for a prolonged period of time.

The short synacthen test (SST) is the most commonly performed investigation to assess adrenal function $(1,2)$ as it is more practical than the 'gold standard' insulin tolerance test (ITT) (3).

In some, but far from all centres a 60-minute serum cortisol post-synacthen is still checked as part of the STT protocol, while in other centres it is not deemed necessary to do this. In the light of these differences in practice, we investigated whether the 60-minute serum cortisol adds value to interpretation of the SST.

All patients attended a single centre, Salford Royal Foundation Trust (SRFT) for their tests. Data from 207 consecutive SSTs were obtained from the Hospital Electronic Patient Record (EPR). All tests were performed before midday. We looked at a period of 12 months from mid-June 2017 to mid-June 2018. Where a patient had more than one SST, the first of the SSTs was analysed.

Serum cortisol was measured by immunoassay on the Siemens Centaur XP analyser (Erlangen, Germany). The analytical range of this assay is $13.8-2069 \mathrm{nmol} / \mathrm{L}$.

Whenever possible, an individual on oral corticosteroids was changed to oral hydrocortisone for 48 hours prior to the SST being performed (if they were not already on this) with omission of hydrocortisone on the evening before and the morning of the test or omission on the morning of the test if on daily prednisolone/dexamethasone. A 30-60-minute cortisol concentration of [?]450nmol/L defined a pass; 350-449nmol/L defined borderline.

Our findings were that in relation to the laboratory assay post-synacthen cortisol pass cut-off of [?]450nmol/L, in $16 / 207(7.2 \%)$ of cases the 60-minute cortisol was [?]450nmol/L (adequate adrenocortical function) but the 30-minute cortisol was below this. Importantly in all cases where the 30-minute cortisol did indicate a pass (i.e. was [?]450nmol/L) the 60-minute cortisol was also [?]450nmol/L. Thus the 60-minute cortisol measurement resulted in $7.2 \%$ of patients being deemed to have adequate adrenocortical function when the 30-minute cortisol would not have done so.

The 60-minute cortisol therefore retains utility in ruling out adrenocortical insufficiency. Determination of the 60-minute cortisol is done in some endocrine centres and was supported by the work of Chitale et al (4). 
The authors of that paper stated that individuals passing the SST only at 60 min tend to exhibit a 'delayed response' to exogenous adrenocorticotrophic hormone (ACTH) but in essence have normally functioning adrenal glands. This conclusion was supported in a recent paper by Butt et al (5) who looked at 849 people undergoing SSTs and reported that $9.5 \%$ of patients had a suboptimal response at 30 minutes, but reached the threshold value at 60 minutes. There is also the possibility that the 60 -minute serum cortisol level may actually be more representative of physiological adrenocortical function than the 30-minute serum cortisol (6).

If patient management is based solely on the 30 -min sample, the individual have to undergo further tests or may be commenced on unnecessary, long-term steroid replacement therapy.

To conclude, we propose that the 60-minute cortisol should be part of the SST as relying on 30-minute cortisol level alone may falsely ascribe individuals to having adrenocortical insufficiency. It would surely be sensible to work towards an international consensus in relation to whether or not the 60-minute cortisol should be part of all SST protocols.

Acknowledgements

The authors thank Dr Lewis Green (Salford Royal Foundation Trust) assisting with data collation.

\section{Transparency statement}

Dr. Heald as corresponding author affirms that this is an honest, accurate, and transparent account of the study being reported; that no important aspects of the study have been omitted; and that any discrepancies from the study as planned (and, if relevant, registered) have been explained.

\section{Ethics Statement}

As this project was deemed by our local Research and Development Committee as a quality improvement (QI) project, no individual patient was contacted in the course of the evaluation and data was fully anonymised prior to analysis, it was not felt that formal ethics permission was required.

\section{Funding}

We have no external funding to declare.

\section{Role of the Sponsor}

There was no research sponsor for this study

\section{Dissemination of study results to participants}

Dissemination to interested patient groups both locally and nationally will be done once the analysis has been finalised

\section{Patient Consent}

This was not deemed necessary as no individual patient was contacted or asked to do anything beyond their usual clinical care.

\section{Data Availability}

We used patient level data which was fully anonymised prior to analysis. Any requests for access to this data should be made to the corresponding author, Dr Adrian Heald. 


\section{Duality of interest}

No author has anything to disclose in relation to conflict of interest.

\section{Contribution Statement}

AHH and AR conceived the study. MM and LM collected the data. MM, AAF and GY conducted the data analysis. CJD, ML and AAF provided perspective from the laboratory. AN assisted with the literature review.

MM, LM, GY, ML, SD, AR, AN, AAF, CJD, PT and AHH all contributed to writing of the paper. AAF, and PT provided an overview of the manuscript.

\section{References}

1. Grossman AB. Clinical review: The diagnosis and management of central hypoadrenalism. J Clin Endocrinol Metab 2010; 95: 4855-4863

2. Hanna FWF, Issa BG, Keevil B et al. Investigating cortisol excess or deficiency: a practical approach. BMJ. 2019; 367: 16039.

3. Jones SL, Trainer PJ, Perry L et al. An audit of the insulin tolerance test in adult subjects in an acute investigation unit over one year. Clin Endocrinol (Oxf) 1994; 41: 123-128

4. Chitale A, Musonda P, McGregor AM et al. Determining the utility of the 60 min cortisol measurement in the short synacthen test. Clin Endocrinol (Oxf) 2013; 79: 14-9

5. Butt MI, Alzuhayri N, Amer L, Riazuddin M, Aljamei H, Khan MS, Abufarhaneh M, Alrajhi E, Alnassar A, Alahmed R, Aljayar DMA, Abothenain FF, De Vol E. Comparing the utility of 30- and 60-minute cortisol levels after the standard short synacthen test to determine adrenal insufficiency: A retrospective cross-sectional study. Medicine (Baltimore) 2020; 99: e22621

6. Zueger T, Jordi M, Laimer M, Stettler C. Utility of 30 and 60 minute cortisol samples after high-dose synthetic ACTH-1-24 injection in the diagnosis of adrenal insufficiency. Swiss Med Wkly 2014; 144: w13987. doi: $10.4414 / \mathrm{smw} .2014 .13987$ 